\title{
Perspective effects in repeated reading: An eye movement study
}

\author{
JoHANNA K. KAAKINEN AND JUKKA HYÖNÄ \\ University of Turku, Turku, Finland
}

\begin{abstract}
The present study examined the influence of perspective instructions on online processing of expository text during repeated reading. Sixty-two participants read either a high or a low prior knowledge (HPK vs. LPK) text twice from a given perspective while their eye movements were recorded. They switched perspective before a third reading. Reading perspective affected the first-pass reading and also increased sentence wrap-up processing time in the perspective-relevant sentences. Prior knowledge facilitated the recognition of the (ir)relevance of text information and resulted in relatively earlier perspective effects in the HPK versus LPK text. Repeated reading facilitated processing, as indicated by all eye movement measures. After the perspective switch, a repetition benefit was observed for the previously relevant text information, whereas a repetition cost was found for the previously irrelevant text information. These results indicate that reading perspective and prior knowledge have a significant influence on how readers allocate visual attention during reading.
\end{abstract}

The present study examined the influence of reading perspective on the online processing of expository texts. Reading perspective refers to the vantage point or the mental frame from which the reader approaches a text. For example, when you are reading a travel guide in order to find information about a specific country - say, Finland - it can be said that you have a specific reading perspective in mind. The perspective makes certain facts in the text more important or relevant to the reader than others (e.g., Pichert \& Anderson, 1977). Memory studies suggest that reading perspective affects the encoding of text information: After reading, readers recall more perspective-relevant than other text information (e.g., Anderson, Pichert, \& Shirey, 1983; Baillet \& Keenan, 1986; but see Anderson \& Pichert, 1978), and changing the perspective at the time of recall does not help in recalling new facts from the text (e.g., Anderson et al., 1983; Baillet \& Keenan, 1986; Kaakinen, Hyönä, \& Keenan, 2001).

According to the current theories of text comprehension, top-down factors such as reading perspective exert their influence relatively late in the course of text processing (e.g., Kintsch, 1998). For example, according to Kintsch's $(1988,1998)$ construction-integration (CI) theory, the text comprehension process proceeds in a twophase cycle. First, during the construction phase, the text input launches a dumb bottom-up process in the reader's knowledge base. All concepts associated with the input in the reader's knowledge base become activated. Next, in the integration phase, the activation in the knowledge network stabilizes by a constraint satisfaction process: Those concepts that fit in with the text and with each other are retained, but other concepts become deactivated. As sen- tences are read, new propositions are integrated into the developing text representation via this kind of CI process. The resulting memory representation contains both text information and information retrieved from the reader's knowledge base. In the CI model, top-down factors, such as reading perspective or reading goal, exert their influence at the integration phase. Moreover, applying Kintsch's CI model to perspective-driven text processing, it can be assumed that only perspective-relevant text information is fully integrated into the developing memory representation, whereas perspective-irrelevant text information is processed only as far as to recognize its irrelevance, and thus, it is only roughly encoded to memory.

Previous online studies have shown that readers do adjust their online reading strategies so that they spend longer time viewing relevant facts than irrelevant text information (e.g., Goetz, Schallert, Reynolds, \& Radin, 1983; Kaakinen, Hyönä, \& Keenan, 2002). A think-aloud study of Kaakinen and Hyönä (2005) further demonstrates that the extra time spent on relevant text information is used to rehearse relevant information in order to encode it to memory. However, the existing evidence is indecisive as to whether reading perspective exerts its effect only on the integration phase or whether reading perspective already affects the construction phase (i.e., when the text input is first encountered). Even though, in the CI model, top-down effects can influence only the integration phase, it is possible that perspective also modifies the scanning strategies adopted during the first-pass reading of text. In this case, the reading perspective would influence the construction phase by defining what type of text input is processed to a deeper level. 
Prior knowledge is a critical factor in determining how easily text information is encoded to memory (e.g., Kintsch \& Franzke, 1995; McNamara \& Kintsch, 1996; Moravcsik \& Kintsch, 1995; Voss \& Silfies, 1996). According to the long-term working memory (LT-WM) model of Ericsson and Kintsch (1995), text input serves as a retrieval cue for concepts in long-term memory: A text cue can make available a whole subset of information in the reader's knowledge base. When a reader has ample prior knowledge related to the text contents, LT-WM provides fast access to relevant knowledge in the reader's knowledge base, enabling the construction of a comprehensive representation of a text (Kintsch, Patel, \& Ericsson, 1999). Encoding perspective-relevant text information to memory should thus be easier and require only little additional processing time if readers have ample prior knowledge of the text contents than when little or no prior knowledge is available. In accordance with this view, recent evidence suggests that the influence of a reading perspective on online text processing is modulated by the amount of prior knowledge the reader has about the relevant text contents. In the study of Kaakinen et al. (2003), participants read two expository texts, one describing familiar and one unfamiliar diseases, while their eye movements were recorded. Before reading, the participants were instructed to imagine that they would need to tell other people things about one of the diseases described in the text. The results showed that when reading the low prior knowledge (LPK) text (unfamiliar diseases), readers spent longer time reading sentences describing the relevant illness than sentences providing information about a perspective-irrelevant disease. In the high prior knowledge (HPK) text (familiar diseases), on the other hand, the perspective effect on online processing was significantly reduced. Yet memory performance was better for the relevant than for the irrelevant text information in both LPK and HPK conditions. Kaakinen et al. (2003) suggested that prior knowledge helps in assigning relevance to text information and in encoding it to memory, thus reducing the perspective effect in processing (i.e., the difference in reading time between perspective-relevant and perspective-irrelevant sentences).

Even though previous research shows that a reading perspective has a great impact on how readers process and remember text information, the exact time course and nature of the perspective effects are still unknown. The exact time course is unknown because, in previous studies (Kaakinen \& Hyönä, 2005; Kaakinen et al., 2002, 2003), perspective effects have been analyzed using sentence-level measures of eye movements. In the present study, we employed word-level analyses to obtain a more detailed understanding of how reading perspective is utilized during online text processing. Moreover, previous studies have examined perspective effects only during a single reading of a text. However, it is possible that the nature of the perspective effects changes when the text is reread. In the present study, we examined perspective effects when readers reread the same text. The nature of the perspective effects on text memory has previously been studied using only text recall. In the present experiment, we also utilized the repeated reading paradigm and perspective shift instructions to examine the memory representation constructed for relevant and irrelevant text information. In sum, the present study aimed at answering three questions: (1) What is the exact time course of perspective effects? (2) How is reading perspective utilized when text is reread? And (3) What is the quality of memory representation constructed for relevant and irrelevant text information? Because previous research suggests that prior knowledge modulates perspective effects on text comprehension, the above-mentioned questions were studied using a text of familiar contents and another of unfamiliar contents.

The following predictions were made regarding the time course of perspective effects. If reading perspective influences online text processing very early on, gaze duration (first-pass fixation time) should be generally longer when words are read in perspective-relevant sentences than when they are read in perspective-irrelevant sentences. In terms of Kintsch's $(1988,1998)$ CI model, this would mean that reading perspective affects the construction phase. An early effect of reading perspective would also be supported by the data on the probability of skipping over words during the first-pass reading of target sentences. The probability of skipping should be smaller in relevant than in irrelevant sentences. Alternatively, reading perspective may not exert its effect on first-pass reading of words but may primarily affect the integration phase. If so, no perspective effects should be observed until the reader has reached the sentence end. Thus, only the gaze duration of sentence-final words will be longer for relevant than for irrelevant sentences (the so-called sentence wrap-up effect; see, e.g., Just \& Carpenter, 1980; Rayner, Kambe, $\&$ Duffy, 2000). Moreover, if reading perspective affects the integration phase, more regressive fixations will be made when relevant sentences are read than when irrelevant ones are. The regressions may most likely be initiated from the sentence end (Hyönä, 1995). These rereading fixations are assumed to serve the purpose of reprocessing relevant information and suspend the intake of new text information before the currently processed information is encoded to memory to a sufficient degree (see Blanchard \& Iran-Nejad, 1987).

Prior knowledge may modulate the time course of the observed perspective effect. Kaakinen et al. (2003) suggested that prior knowledge helps in assigning relevance to text information and in encoding relevant information to memory. If that is true, we should observe that in the HPK condition, readers recognize the perspective relevance of text information already in the beginning of the sentence, resulting in an early perspective effect. Moreover, the perspective effect may level out toward the end of the sentence. In the LPK condition, however, the perspective effect may "build up" as the reader proceeds in the text and may not appear until the reader has proceeded to the end of the sentence (i.e., it may materialize primarily as a sentence wrap-up effect).

As was pointed out above, we examined in the present study how reading perspective influences online text processing when the same text is reread. Repeated reading of a text typically produces rereading benefits; that is, it speeds up the subsequent reading and enhances com- 
prehension (see Raney, 2003, for a review). Just as prior knowledge may modulate the timing of the perspective effects, prior exposure to the text should also have an impact on how early perspective effects emerge. Perspective effects should show up earlier when readers reread the text than during the first reading, because readers can make use of the memory representation gained during the initial reading to recognize relevant and irrelevant information in the text.

As for the effects of repeated reading on the magnitude of the perspective effects, three possibilities exist. First, it is possible that readers change their processing strategy across the readings, which would result in an increase in the magnitude of the perspective effect from one reading to another (see Millis \& King, 2001; Millis, King, \& Kim, 2000; Millis \& Simon, 1994; Millis, Simon, \& tenBroek, 1998; Shebilske \& Fisher, 1981; Stine-Morrow, Gagne, Morrow, \& DeWall, 2004). According to this view, readers form a general overview of the text contents during the first reading. However, during rereading readers shift to more strategic processing. In the case of perspective-driven reading, it would mean that readers attend selectively to relevant text information and ignore irrelevant information. This would result in significant repetition benefits (speed-up) in the processing of perspective-irrelevant information but significant repetition costs (slow-down) for perspective-relevant text information.

A second possibility is that the magnitude of the perspective effect decreases across the readings. According to this view, readers apply a selective processing strategy already during the initial reading of the text. Due to selectivity in processing during the initial reading, the resulting text representation contains more perspective-relevant than perspective-irrelevant text information. Because perspective-relevant text information is already encoded to memory during the initial reading, the processing of perspective-relevant text information is facilitated during rereading, resulting in a decrease in the magnitude of the observed perspective effect during subsequent readings (see Levy et al., 1995; Levy \& Kirsner, 1989; Raney, 2003). In other words, there should be significant repetition benefits for perspective-relevant text information, whereas the processing of perspective-irrelevant text information is affected by repetition only a little.

Finally, the results of Hyönä and Niemi (1990) suggest yet another possibility - namely, that relevance of text information is such a strong factor in controlling the allocation of processing resources that the magnitude of the perspective effect does not change across readings. Thus, repetition benefits would be of equal magnitude for relevant and irrelevant text information.

In the present study, the third reading of the same text was done from a different perspective. This means that text information that was relevant during the first two readings now becomes irrelevant and text information that was irrelevant during the first readings is now relevant when assessed from the new reading perspective. If the initially perspective-irrelevant information is encoded as part of the text representation during the first readings, processing of the same information as perspective relevant during a subsequent reading should lead to a repetition benefit or, at least, should not result in a reprocessing cost. However, this may not be very likely. Previous studies have demonstrated a relatively poor memory for perspective-irrelevant text information. Thus, it is more likely that readers spend the extra time processing the perspective-relevant information that was initially processed as perspective irrelevant. On the other hand, when text information is encoded to memory as perspective relevant during the first readings, its reprocessing as perspective-irrelevant information during the third reading should lead to a substantial repetition benefit.

In the present study, participants read either an LPK text describing rare diseases or an HPK text describing familiar illnesses (Kaakinen et al., 2003). A reading perspective was induced by introducing a scenario in which the participants would need to tell other people about one of the diseases described in the text. Readers read the texts twice from the same perspective, but between the second and the third reading, they were instructed to switch perspective. For example, if the participant had been instructed to imagine that he or she would need to tell other people things about trigeminusneuralgy, he or she was now instructed to imagine that he or she would need to tell other people things about typhus (a disease that was irrelevant during the initial reading). Words in the target sentences were grouped into beginning, middle, and final words. We then analyzed the fixation time on words during the first-pass reading (gaze duration), the probability of skipping a word (i.e., leaving a word unfixated during the first-pass reading), and the probability of making a regression. Gaze duration and the probability of skipping are first-pass reading time measures, whereas the probability of regression reflects reprocessing of sentence contents. After the first two readings, the participants wrote a free recall of the text; after the third reading, they were asked to add details to their recall if they remembered new information.

\section{METHOD}

\section{Participants}

Sixty-two University of Turku students enrolled in an introductory psychology course participated in the experiment. All the participants received course credit for participation.

\section{Apparatus}

Eye movements were collected by the EyeLink II eyetracker, manufactured by SR Research Ltd. (Toronto). The eyetracker is an infrared video-based tracking system combined with hyperacuity image processing. There are two cameras mounted on a headband (one for each eye), including two infrared LEDs for illuminating each eye. The headband weighs $450 \mathrm{~g}$ in total. The cameras sample pupil location and pupil size at the rate of $250 \mathrm{~Hz}$. Registration can be done either monocularly or binocularly. We performed it for the selected eye (usually the right eye) by placing the camera and the two infrared lights 4-6 cm away from the eye. The resolution of eye position is $15^{\prime \prime}$ of arc, and the spatial accuracy is approximately $0.5^{\circ}$. Head position with respect to the computer screen is tracked with the help of a head-tracking camera mounted on the center of the headband at the level of the forehead. Four LEDs are attached to the corners of the computer screen, which are viewed by the headtracking camera once the participant sits directly facing the screen. 
Possible head motion is detected as movements of the four LEDs and is compensated for online from the eye position records.

\section{Materials}

The experimental texts were adopted from the study of Kaakinen et al. (2003). Both texts were expository texts written to comply with a compare-contrast rhetorical structure. The rare diseases text was 878 words long and discussed four infrequent diseases: trigeminusneuralgy, typhus, cystic fibrosis, and scleroderma. The participants had very little knowledge of these diseases before reading the text (for familiarity ratings, see Kaakinen et al., 2003). The familiar diseases text was 1,065 words long and described four illnesses familiar to the participants: flu, diarrhea, chicken pox, and AIDS. The text contents were subsumed into subtopics, each marked with a subheading, and the diseases were presented under each subtopic by comparing and contrasting the diseases with each other (see the Appendix for an example paragraph for both texts). The texts were written in Finnish, the native language of the study participants. Half of the participants read the rare diseases text, and half read the familiar diseases text.

For both texts, there were two possible reading perspectives that could be adopted. One of the perspectives was assigned to each participant before the initial reading. In the rare diseases text, the reading perspective was induced by instructing the participant to

imagine that a close friend of yours has been diagnosed with trigeminusneuralgy/typhus. Everybody is very worried about this common friend, and you have agreed to find out some facts about the disease and to inform the others about it. Read the text in order to be able to do the job.

In the familiar diseases text, the perspective was induced by instructing the participant to

imagine that you are going to give a health education class for elementary school pupils. You are supposed to tell them about flu/diarrhea: for example, what causes it, how you can treat it, and how to prevent from getting the disease.

Both texts included 20 preselected target sentences, 10 sentences discussing each of the two target diseases. As can be seen in the sample paragraphs in the Appendix, not all potentially relevant (or irrelevant) sentences in the text were included in the analyses. The selection of the 10 relevant target sentences for each disease was dictated by two criteria: (1) The sentence was clearly relevant to the disease in question, and (2) it matched as closely as possible (in length and in the frequency of the words) with a target sentence in the other information sets (frequencies were calculated from an unpublished Finnish newspaper corpus with the help of the WordMill database program of Laine \& Virtanen, 1999). ${ }^{1}$ Since the reading perspective was counterbalanced across participants, for the half of the participants reading the rare diseases text, the trigeminusneuralgy-relevant target sentences were the perspective-relevant sentences, and the typhus-relevant target sentences were the perspective-irrelevant sentences, whereas for the other half, the typhus-relevant sentences were perspective relevant, and the trigeminusneuralgy-relevant sentences were perspective irrelevant. An analogous counterbalancing was done for the familiar diseases text.

\section{Procedure}

The participants were seated approximately $60 \mathrm{~cm}$ from the computer screen. Prior to the experiment, the eyetracker was adjusted and calibrated using a 9-point calibration grid that extended over the entire computer screen. The participants were informed that they were going to read an expository text twice in succession. Written instructions, in which the reading perspective was given, were presented on the screen before the experimental text. The text was presented on a 17 -in. monitor with a $640 \times 480$ resolution. The line spacing was set to 2.8 , which allowed eight lines of text to be presented on one screen. The participant proceeded in the text by pressing a button on a game pad. Immediately after the first reading, the eyetracker was recalibrated, after which the participants read the text for the second time. After reading the text twice, the participants were given the reading perspective they had adopted and the title of the text as the recall cues. The participants were instructed to write down everything they could remember of the text, not just what was related to the title. After completing the recall, the participants returned for the third reading. They were instructed to shift perspective, and new perspective instructions were presented prior to the third reading. If they had read the LPK text from the trigeminusneuralgy perspective, they were now presented the typhus perspective, and vice versa. Similarly, if they had read the HPK text from the flu perspective, they were now given the diarrhea perspective, and vice versa. After the third reading, they were asked to add to the previously written recall protocol whatever information they now recalled that they had omitted from the initial recall; in addition, they were asked to correct mistakes in their protocol if they noticed any. The participants were allowed to leave whenever they were ready. The entire session lasted about $1.5 \mathrm{~h}$

\section{RESULTS}

Data for 2 participants had to be excluded due to problems with the calibration of the eyetracker. To examine the time course of processing, words in the target sentences were categorized into three regions. The first two words of a sentence were regarded as the beginning of the sentence, and the last two words as the end of the sentence. All other words were coded as sentence-middle words. For target sentences consisting of only four words, data were included only for the beginning and end parts of the sentence.

Three different processing measures were computed from the eye fixation data: gaze duration (i.e., summed duration of fixations prior to exiting a word for the first time), probability of skipping a word (i.e., leaving a word unfixated), and probability of making a regression during target sentence reading. The first two measures are first-pass processing measures, whereas the last one reflects the second-pass reading of words. Each measure was first computed separately for each word in the target sentences. Then, on the basis of these data, separate means were computed for the beginning, middle, and end parts of the relevant and irrelevant target sentences. In the following, we first will report the analyses of the initial reading of the text in order to examine the detailed time course of the perspective effects when the target sentences were encountered for the first time. We then will report the analyses of repetition effects observed in the second reading, followed by repetition effects observed after a new perspective was adopted before the third reading. We will close the Results section by presenting the recall data.

\section{Time Course of the Perspective Effects During the Initial Reading of the Text}

To analyze the time course of perspective effects, we computed separate 2 (text: LPK vs. HPK) $\times 2$ (perspective relevance: relevant vs. irrelevant) $\times 3$ (position: beginning, middle, or end of the sentence) repeated measures ANOVAs for all three eye movement measures. Text was the only between-participants factor. Greenhouse-Geisser corrections to the degrees of freedom were applied whenever the sphericity assumption was violated. 
Table 1

\begin{tabular}{|c|c|c|c|c|c|c|c|c|}
\hline $\begin{array}{r}\text { Mean Ga } \\
\text { in the } R \\
\text { Fir }\end{array}$ & $\begin{array}{l}\text { Dur } \\
\text { van } \\
\text { Seco } \\
\text { unc }\end{array}$ & $\begin{array}{l}\mathbf{a} \\
\mathbf{0}\end{array}$ & $\begin{array}{l}\text { Mil } \\
\text { eva } \\
\text { hir } \\
\text { rd }\end{array}$ & & $\begin{array}{l}\text { Sen } \\
\text { s of } \\
\text { in T }\end{array}$ & $\begin{array}{l}\text { Te } \\
\text { Ty }\end{array}$ & ing & \\
\hline & & Kn & dge & & & $\begin{array}{r}\mathrm{I} \\
\mathrm{Kn}\end{array}$ & edge & \\
\hline Worc & $\begin{array}{l}\text { Rel } \\
\text { Sen }\end{array}$ & $\begin{array}{l}\text { ant } \\
\text { ces }\end{array}$ & $\begin{array}{l}\text { Irre } \\
\text { Sen }\end{array}$ & & $\begin{array}{l}\text { Rel } \\
\text { Sen }\end{array}$ & $\begin{array}{l}\text { int } \\
\text { ces }\end{array}$ & $\begin{array}{l}\text { Irre } \\
\text { Sen }\end{array}$ & $\begin{array}{l}\text { ant } \\
\text { ces }\end{array}$ \\
\hline Position & $M$ & $S E$ & $M$ & $S E$ & $M$ & $S E$ & $M$ & $S E$ \\
\hline & & & First & din & & & & \\
\hline Beginning & 334 & 13 & 308 & 13 & 282 & 13 & 264 & 13 \\
\hline Middle & 303 & 11 & 278 & 9 & 262 & 11 & 235 & 9 \\
\hline End & 444 & 20 & 389 & 20 & 346 & 20 & 296 & 20 \\
\hline & & & $\mathrm{col}$ & an & & & & \\
\hline Beginning & 311 & 14 & 291 & 18 & 262 & 10 & 248 & 10 \\
\hline Middle & 280 & 8 & 259 & 10 & 257 & 10 & 228 & 8 \\
\hline End & 414 & 26 & 347 & 21 & 307 & 12 & 271 & 14 \\
\hline & & & hird & ad11 & & & & \\
\hline Beginning & 306 & 12 & 268 & 13 & 254 & 8 & 238 & 7 \\
\hline Middle & 274 & 8 & 249 & 10 & 234 & 5 & 221 & 7 \\
\hline End & 388 & 20 & 317 & 17 & 330 & 13 & 253 & 11 \\
\hline
\end{tabular}

Gaze duration. If reading perspective exerts its influence already during the initial construction phase (i.e., when sentence meaning is extracted) by modifying the scanning strategies adopted by the reader, we should observe longer gaze durations for words in the relevant sentences than for those in the irrelevant target sentences already before the sentence end was reached. However, if reading perspective exerts its influence mainly at the integration phase, the perspective effect should be localized in the sentence end (i.e., as a sentence wrap-up effect). We also expected that the perspective effect would manifest itself earlier in the HPK than in the LPK text.

Words in the relevant sentences attracted longer gaze durations than did words in the irrelevant sentences, as indicated by a main effect of relevance $[F(1,58)=24.37$, $\left.M S_{\mathrm{e}}=4,166.53, \eta^{2}=.30\right]$. A significant interaction between word position and relevance $[F(2,100)=3.53$, $\left.M S_{\mathrm{e}}=2,672.80, \eta^{2}=.06\right]$ suggests that the magnitude of the perspective effect in gaze duration depended on the position of the word in the sentence. As is evident from Table 1, the difference between relevant and irrelevant sentences was $22 \mathrm{msec}$ in the beginning, $26 \mathrm{msec}$ in the middle, and $52 \mathrm{msec}$ in the end of the sentences. All these differences were significant [smallest $t(59)=2.55, S E=$ 8.72 , for the beginning], but the perspective effect was strongest at the sentence end.

Also, significant main effects of position $[F(2,88)=$ $\left.62.90, M S_{\mathrm{e}}=6,706.91, \eta^{2}=.52\right]$ and text $[F(1,58)=$ $\left.15.29, M S_{\mathrm{e}}=22,601.44, \eta^{2}=.21\right]$ were observed, as well as a significant position $\times$ text interaction $[F(2,88)=$ 5.03, $\left.M S_{\mathrm{e}}=6,706.91, \eta^{2}=.08\right]$. The position effect reflects the fact that gaze durations were longest for the sentence-final words $(369 \mathrm{msec})$ and shortest for the sentence-middle words $(270 \mathrm{msec})$. The main effect of text is a result of the average gaze duration's being $62 \mathrm{msec}$ longer on words in the LPK text than on those in the HPK text. Finally, the position $\times$ text interaction reflects the finding that the two texts differed particularly in the gaze duration for the sentence-final words (see Table 1).

In sum, reading perspective affected gaze duration already for the first two words of the target sentence. However, the impact of reading perspective was especially strong in the end of the target sentences, suggesting that reading perspective increases the need for integrative sentence wrap-up processing of perspective-relevant information. Moreover, the overall wrap-up effect was more pronounced for the LPK text than for the HPK text. On the other hand, the gaze duration data did not support the prediction that the perspective effect would show up earlier in the text of familiar contents (however, see the Probability of Skipping section below).

Probability of skipping. If readers modify their scanning strategies on the basis of the adopted reading perspective and the perspective thus exerts its influence during the first encounter with the target information, the probability of skipping should be higher for words in the irrelevant sentences than for those in the relevant sentences. Moreover, if prior knowledge modulates the perspective effects, the perspective effect should occur earlier in the HPK text than in the LPK text.

Readers skipped more words in the irrelevant sentences than in the relevant sentences $\left[F(1,58)=20.01, M S_{\mathrm{e}}=\right.$ $\left.0.01, \eta^{2}=.26\right]$. However, a significant interaction between relevance and text $\left[F(1,58)=7.70, M S_{\mathrm{e}}=0.01, \eta^{2}=.12\right]$ suggests that the perspective effect was different in the two texts, and hence, separate analyses of the two texts were computed. The only significant effect in the LPK text was the main effect of position $\left[F(2,46)=70.33, M S_{\mathrm{e}}=0.35\right.$, $\left.\eta^{2}=.71\right]$. Sentence-middle words were skipped the most, and sentence-final words the least (see Table 2). However, the main effect of relevance failed to reach significance $\left[F(1,29)=3.28, M S_{\mathrm{e}}=0.005, p=.08, \eta^{2}=.10\right]$, indicating that even though there was a tendency toward more

Table 2

Mean Probabilities of Skipping (With Standard Errors) in the Relevant and Irrelevant Target Sentences During the First, Second, and Third Readings of the Text As a Function of Word Position and Text Type

\begin{tabular}{|c|c|c|c|c|c|c|c|c|}
\hline \multirow{3}{*}{$\begin{array}{c}\text { Word } \\
\text { Position }\end{array}$} & \multicolumn{4}{|c|}{$\begin{array}{c}\text { Low } \\
\text { Prior Knowledge Text }\end{array}$} & \multicolumn{4}{|c|}{$\begin{array}{c}\text { High } \\
\text { Prior Knowledge Text }\end{array}$} \\
\hline & \multicolumn{2}{|c|}{$\begin{array}{l}\text { Relevant } \\
\text { Sentences }\end{array}$} & \multicolumn{2}{|c|}{$\begin{array}{l}\text { Irrelevant } \\
\text { Sentences }\end{array}$} & \multicolumn{2}{|c|}{$\begin{array}{l}\text { Relevant } \\
\text { Sentences }\end{array}$} & \multicolumn{2}{|c|}{$\begin{array}{l}\text { Irrelevant } \\
\text { Sentences }\end{array}$} \\
\hline & $M$ & $S E$ & $M$ & $S E$ & $M$ & $S E$ & $M$ & $S E$ \\
\hline \multicolumn{9}{|c|}{ First Reading } \\
\hline Beginning & .17 & .02 & .17 & .02 & .16 & .02 & .23 & .02 \\
\hline Middle & .21 & .02 & .25 & .02 & .28 & .02 & .35 & .02 \\
\hline End & .08 & .01 & .10 & .03 & .21 & .01 & .31 & .03 \\
\hline \multicolumn{9}{|c|}{ Second Reading } \\
\hline Beginning & .20 & .02 & .21 & .02 & .24 & .03 & .29 & .03 \\
\hline Middle & .24 & .02 & .28 & .02 & .35 & .02 & .43 & .03 \\
\hline End & .10 & .01 & .14 & .02 & .29 & .03 & .39 & .04 \\
\hline \multicolumn{9}{|c|}{ Third Reading } \\
\hline Beginning & .19 & .02 & .24 & .03 & .24 & .02 & .29 & .03 \\
\hline Middle & .26 & .01 & .33 & .03 & .34 & .02 & .45 & .03 \\
\hline End & .11 & .02 & .18 & .03 & .26 & .02 & .44 & .04 \\
\hline
\end{tabular}


Table 3

Mean Probabilities of Making a Regression (With Standard Errors) in the Relevant and Irrelevant Target Sentences During the First, Second, and Third Readings As a

Function of Word Position and Text Type

\begin{tabular}{|c|c|c|c|c|c|c|c|c|}
\hline \multirow{3}{*}{$\begin{array}{l}\text { Word } \\
\text { Position }\end{array}$} & \multicolumn{4}{|c|}{$\begin{array}{c}\text { Low } \\
\text { Prior Knowledge Text }\end{array}$} & \multicolumn{4}{|c|}{$\begin{array}{c}\text { High } \\
\text { Prior Knowledge Text }\end{array}$} \\
\hline & \multicolumn{2}{|c|}{$\begin{array}{l}\text { Relevant } \\
\text { Sentences }\end{array}$} & \multicolumn{2}{|c|}{$\begin{array}{l}\text { Irrelevant } \\
\text { Sentences } \\
\end{array}$} & \multicolumn{2}{|c|}{$\begin{array}{c}\text { Relevant } \\
\text { Sentences } \\
\end{array}$} & \multicolumn{2}{|c|}{$\begin{array}{l}\text { Irrelevant } \\
\text { Sentences }\end{array}$} \\
\hline & $M$ & $\overline{S E}$ & $M$ & $\overline{S E}$ & $M$ & $\overline{S E}$ & $M$ & $\overline{S E}$ \\
\hline \multicolumn{9}{|c|}{ First Reading } \\
\hline Beginning & .13 & .04 & .10 & .03 & .21 & .04 & .13 & .03 \\
\hline Middle & .15 & .02 & .09 & .01 & .09 & .02 & .08 & .01 \\
\hline End & .33 & .03 & .19 & .03 & .20 & .03 & .17 & .03 \\
\hline \multicolumn{9}{|c|}{ Second Reading } \\
\hline Beginning & .13 & .02 & .08 & .02 & .17 & .03 & .09 & .03 \\
\hline Middle & .11 & .01 & .06 & .01 & .07 & .01 & .06 & .01 \\
\hline End & .28 & .03 & .12 & .02 & .14 & .02 & .09 & .02 \\
\hline \multicolumn{9}{|c|}{ Third Reading } \\
\hline Beginning & .09 & .02 & .10 & .02 & .16 & .03 & .09 & .02 \\
\hline Middle & .16 & .02 & .07 & .01 & .09 & .02 & .04 & .01 \\
\hline End & .32 & .04 & .13 & .02 & .22 & .03 & .08 & .01 \\
\hline
\end{tabular}

skipping of words in the irrelevant than in the relevant sentences (.17 in the irrelevant vs. 15 in the relevant), readers were reluctant to skip words in the irrelevant sentences when they had very little prior knowledge of the text contents. In the HPK text, on the other hand, readers skipped more words in the irrelevant than in the relevant sentences [.30 in the irrelevant vs. .22 in the relevant; $F(1,29)=$ $\left.16.84, M S_{\mathrm{e}}=.02, \eta^{2}=.37\right]$. Also, the main effect of position was significant $\left[F(1,42)=30.24, M S_{\mathrm{e}}=0.01, \eta^{2}=\right.$ .51]. Sentence-middle words were skipped the most, and sentence-initial words the least.

Other effects observed in the omnibus analysis were a significant main effect of position $[F(2,89)=63.87$, $\left.M S_{\mathrm{e}}=0.01, \eta^{2}=.52\right]$ and an interaction between position and text $\left[F(2,89)=26.46, M S_{\mathrm{e}}=0.01, \eta^{2}=.31\right]$. The two texts differed notably in that readers skipped over words more in the HPK than in the LPK text in the middle of the sentence [.32 vs. .23; $t(58)=3.63, S E=.02]$ and particularly in the end of the sentence $[.26$ vs. $.09 ; t(58)=$ $6.12, S E=.03]$.

The results on the probability of skipping indicate that prior knowledge modulated the observed perspective effect; readers used a more selective strategy in the HPK text than in the LPK text during the first-pass reading of the target sentences. This became particularly apparent in the higher probability of word skipping in the perspectiveirrelevant sentences.

Probability of regression. Here, we will report the data on the probability of initiating a regressive fixation during target sentence processing. If reading perspective influences the integration phase of text comprehension, we should observe that readers initiate more regressive fixations in the relevant than in the irrelevant sentences, especially from the sentence end. The perspective effect was also expected to show up earlier in the HPK text than in the LPK text.
Readers were more likely to regress in the relevant than in the irrelevant sentences $\left[F(1,58)=23.00, M S_{\mathrm{e}}=0.01\right.$, $\left.\eta^{2}=.28\right]$. A significant interaction between position, relevance, and text $\left[F(2,93)=4.62, M S_{\mathrm{e}}=0.01, \eta^{2}=.07\right]$ indicates that there were differences between the texts in the time course of the perspective effect. The three-way interaction was examined further by separate analyses of the texts.

In the LPK text, readers were more likely to regress in the relevant than in the irrelevant sentences, as indicated by a significant main effect of relevance $[F(1,29)=15.68$, $\left.M S_{\mathrm{e}}=0.02, \eta^{2}=.35\right]$. A significant two-way interaction between relevance and position $\left[F(2,48)=4.18, M S_{\mathrm{e}}=\right.$ $\left.0.01, \eta^{2}=.13\right]$ indicates that the perspective effect was modulated by the position of the word in the sentence (see Table 3). The difference in the probability of regression between relevant and irrelevant sentences was only $3 \%$ in the beginning of the sentence $(t<1)$ and $6 \%$ in the middle of the sentence $[t(29)=4.62, S E=0.01]$ but was as big as $14 \%$ in the end of the sentence $[t(29)=4.63, S E=$ $0.03]$. In general, most regressions were initiated from the end of the sentence, as shown by a main effect of position $\left[F(2,47)=28.99, M S_{\mathrm{e}}=0.02, \eta^{2}=.50\right]$.

Also, in the HPK text, readers were more likely to regress in the relevant than in the irrelevant sentences $\left[F(1,29)=7.41, M S_{\mathrm{e}}=0.01, \eta^{2}=.20\right]$. However, the two-way interaction failed to reach significance $(F<2)$, suggesting that unlike in the LPK text, the magnitude of the perspective effect did not reliably depend on the position of the word in the sentence. However, a closer look at the data revealed that the perspective effect was significant in the sentence beginning [a difference of $8 \%$; $t(29)=2.20, S E=0.04]$, whereas the difference between the relevant and the irrelevant sentences failed to reach significance in the middle $(2 \%)$ or in the end $(3 \%)$ of the sentence (both $t \mathrm{~s}<2$ ). Moreover, as can be seen from Table 3, regressions were typically launched from either the beginning or the end of the target sentence $[F(2,58)=$ $\left.10.07, M S_{\mathrm{e}}=0.02, \eta^{2}=.26\right]$.

Finally, the omnibus analysis also yielded a main effect of position $\left[F(2,103)=28.67, M S_{\mathrm{e}}=0.02, \eta^{2}=.33\right]$, and an interaction between position and text $[F(2,103)=8.56$, $\left.M S_{\mathrm{e}}=0.02, \eta^{2}=.13\right]$. The interaction reflects the finding that more regressions were made from the sentence beginning in the HPK than in the LPK text, whereas more regressions were made from sentence end in the LPK than in the HPK text (however, these differences failed to reach significance in the follow-up analyses). This was primarily the case for the relevant sentences, which suggests that relevance produced an early effect in the HPK text and a later effect in the LPK text.

In sum, the data on the probability of making a regression in the target sentence indicate that readers made more regressions in the perspective-relevant sentences than in the perspective-irrelevant sentences. The timing of regressions differed as a function of the familiarity of text contents; perspective relevance was more likely to induce an early regression in the HPK text and a late regression in the LPK text. 


\section{Repetition Effects During the Second Reading}

We also investigated how the reading perspective was utilized when the readers read the same text twice. In the introduction, we spelled out three different views on how the magnitude of the perspective effect may change across readings. According to one view, readers adopt a selective processing strategy at the second reading. This would result in a significant repetition cost for relevant sentences and a repetition benefit for irrelevant sentences. According to another view, the magnitude of the perspective effect would decrease from the first to the second reading because relevant information is encoded to memory already during the first reading of the text and, thus, the reprocessing of relevant sentences is facilitated. This should lead to significant repetition benefits for relevant sentences but only modest repetition effects for irrelevant sentences. The third alternative is that reading perspective guides online text processing equally strongly across the two readings. In this case, repetition effects would be of similar magnitude for relevant and irrelevant sentences. Finally, we expected the perspective effect to show up earlier during the target sentence processing when those sentences were reread. This was assumed to be especially likely for the HPK text.

In order to define the benefits and costs related to repeated reading of the text, we computed a measure of the repetition effect separately for relevant and irrelevant sentences by using the following formula:

repetition effect $=$ gaze duration/probability of skipping/regression on the first reading - gaze duration/probability of skipping/regression on the second reading

For example, the repetition effect in gaze duration (see Table 1) for the sentence-initial position of the relevant sentences is computed as $334 \mathrm{msec}-311 \mathrm{msec}=$ $23 \mathrm{msec}$. A positive value in gaze duration and in the probability of regression reflects a repetition benefit - that is, faster processing of words during the second reading than during the first reading. A negative value means that processing is slower during the second reading than during the first reading and indicates a processing cost. As for the skipping rate, the opposite is true: A positive value of the repetition effect reflects less skipping (i.e., a cost in processing during the second reading), and a negative value reflects more skipping (i.e., a benefit in processing).

Separate 2 (text: LPK vs. HPK) $\times 2$ (perspective relevance: relevant vs. irrelevant) $\times 3$ (sentence position: beginning, middle, or end) repeated measures ANOVAs were computed for the repetition effects in the second reading. Text was the only between-participants factor. Greenhouse-Geisser corrections to the degrees of freedom were applied when the sphericity assumption was violated.

The only significant effect in gaze duration was the main effect of word position $\left[F(2,88)=4.30, M S_{\mathrm{e}}=4,248.70\right.$, $\left.\eta^{2}=.07\right]$, indicating that in general, largest repetition benefits were observed in the sentence end. The repeti- tion benefits were 19, 14, and $34 \mathrm{msec}$ for the beginning, middle, and end positions, respectively [all these means differed significantly from 0 ; smallest $t(59)=3.48, S E=$ 5.44 , for the beginning]. This may be taken to suggest that repetition particularly facilitated integrative wrap-up processing done at the sentence end prior to the reading of the next sentence. The main effects of relevance and text and all the interactions were not significant (all $\left.F_{\mathrm{S}}<1\right)$. As for the skipping rate, none of the main effects or interactions proved significant (all $F \mathrm{~s}<2$ ). In other words, the magnitude of the repetition effect was not modulated by text relevance, word position, or text type. Finally, for probability of regression, the only significant effect was the main effect of word position $\left[F(2,94)=5.04, M S_{\mathrm{e}}=0.02, \eta^{2}=\right.$ $.08]$, indicating that rereading reduced the need to regress, particularly from the end of the target sentences. This result corroborates the gaze duration results by showing that repetition particularly facilitates integrative wrap-up processing done at the sentence end.

It should be noted that using these difference scores, the main effect of repetition does not show up in the analyses reported above. However, reading the same text again from the same reading perspective brought about a clear facilitation in processing that was seen in all eye movement measures used. Average gaze duration decreased by $22 \mathrm{msec}$ from the first $(312 \mathrm{msec})$ to the second $(290 \mathrm{msec})$ reading, the probability of skipping went up by $5 \%$ (from $21 \%$ to $26 \%$ ), and the probability of regression decreased by $4 \%$ (from $15.6 \%$ to $11.5 \%$ ). All these effects were highly significant $(p<.001)$. To sum up, even though there was a general repetition benefit in processing, the magnitude of the perspective effect remained constant across readings. This result supports the view that relevance of text information is a strong factor in controlling the allocation of processing resources across a repeated exposure to the same text (Hyönä \& Niemi, 1990).

\section{Repetition Effects After a Perspective Change}

To learn more about the exact nature of the perspective effects, we asked the participants to read the text a third time from a different perspective. The relevance of the target sentences was thus changed: Sentences that were relevant during the previous reading became irrelevant, whereas sentences that were irrelevant during the previous reading became relevant after the perspective change. By examining the size of the repetition effects when the same target sentences were read from a different perspective, we hoped to gain further insight into the type of representation readers construct for relevant and irrelevant text information. If the initially perspective-irrelevant information is encoded as part of the text representation during the first two readings, processing of the same information as perspective relevant during the third reading should lead to a repetition benefit or, at least, should not result in a reprocessing cost. However, if the memory representation for originally perspective-irrelevant text information is poor, readers are expected to spend extra time processing the perspective-relevant information (i.e., a repetition cost is predicted). As for the initially relevant 


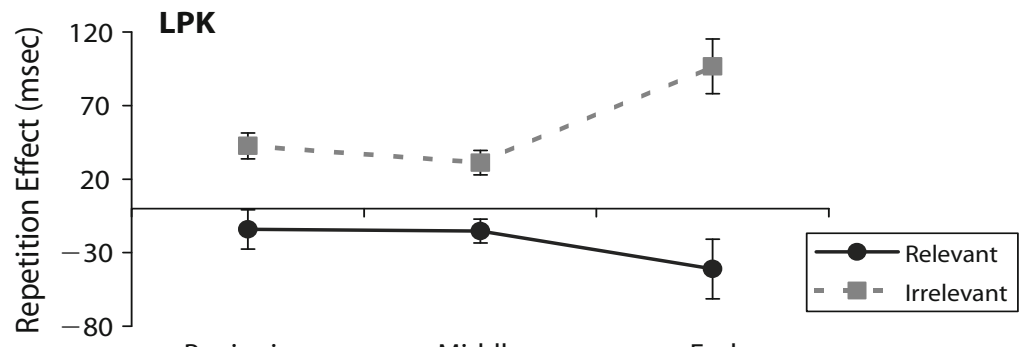

Beginning Middle End

Word Position

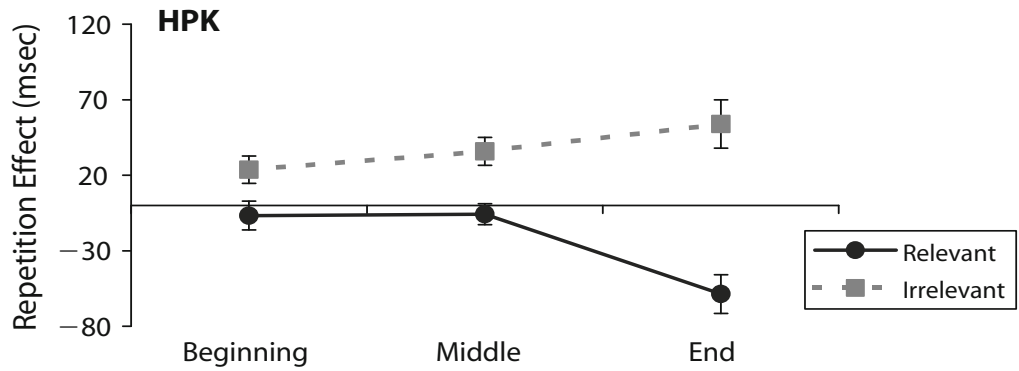

Word Position

Figure 1. Mean repetition effects in gaze duration in the third reading for the relevant and irrelevant text segments as a function of the position of the word in the sentence (error bars represent $1 S E$ above and below the mean). Different panels represent data for different texts: LPK, low prior knowledge text; HPK, high prior knowledge text.

sentences, only one plausible prediction exists: A substantial repetition benefit should be observed when they are subsequently processed as irrelevant.

Repetition effects for relevant and irrelevant sentences were computed with the following formulas:

repetition effect for relevant $=$ gaze duration/ probability of skipping/regression for the irrelevant on the second reading - gaze duration/probability of skipping/regression for the relevant on the third reading

and

repetition effect for irrelevant $=$ gaze duration/ probability of skipping/regression for the relevant on the second reading - gaze duration/probability of skipping/regression for the irrelevant on the third reading.

These measures reflect the repetition effect when the same sentences were previously processed from a different perspective. For example, the repetition effect in gaze duration for the sentence-initial position of the relevant sentences (see Table 1) is computed as $291-306 \mathrm{msec}=$ $-15 \mathrm{msec}$ (see Figure 1). A negative value means that a perspective change caused a repetition cost in the processing of sentences that became relevant. The repetition effect in gaze duration for the sentence-initial position of the irrelevant sentences is computed as $311-268 \mathrm{msec}=$ $43 \mathrm{msec}$ (see Figure 1). A positive value reflects a repetition benefit - that is, a speeding-up of processing of the sentences that became irrelevant after the perspective change. It should be noted that the opposite is true for the skipping rates: A negative value reflects a repetition benefit, and a positive value reflects a repetition cost.

Gaze duration. It should be noted that in the subsequent analyses, perspective relevance is determined by the reading perspective adopted for the third reading. A significant main effect of relevance was observed in gaze duration $\left[F(1,58)=54.83, M S_{\mathrm{e}}=8,256.73, \eta^{2}=.49\right]$. A repetition cost of $24 \mathrm{msec}$ was observed for the relevant sentences, whereas a repetition benefit of $48 \mathrm{msec}$ was observed for irrelevant sentences. Moreover, the relevance $\times$ position interaction proved significant $[F(2,98)=16.55$, $\left.M S_{\mathrm{e}}=4,710.07, \eta^{2}=.22\right]$, indicating that the magnitude of the repetition cost for the relevant sentences and the repetition benefit for the irrelevant sentences changed across the different word positions. In order to examine this interaction in more detail, we computed separate one-sample $t$ tests to examine whether the mean repetition effect differed significantly from 0 for relevant and irrelevant sentences in the different word positions. As is evident from Figure 1, a significant repetition benefit was observed for all word positions of the irrelevant sentences [smallest observed benefit was $33 \mathrm{msec}$ in the sentence beginning; $t(59)=5.17, S E=6.39$ ]. A significant repetition cost for the relevant sentences was observed only for the sentence end [cost of $50 \mathrm{msec} ; t(59)=-4.17, S E=$ 11.97]. Finally, the position $\times$ text interaction $[F(2,93)=$ 3.23, $\left.M S_{\mathrm{e}}=4,123.60, \eta^{2}=.05\right]$ suggests that for the LPK text, there is an overall repetition benefit of $28 \mathrm{msec}$ for 
the sentence-final position, which does not exist for the HPK text (there is a small repetition cost of $3 \mathrm{msec}$ ). This is probably a consequence of the relatively long time used for sentence wrap-up processing during the initial reading of the LPK text.

To sum up the results of the gaze duration data, we observed a repetition benefit for irrelevant sentences. The repetition benefit was particularly pronounced in the sentence-final position, suggesting that repetition facilitated wrap-up processing. On the other hand, a repetition cost was established for relevant sentences, which effect was seen primarily in the sentence-final position. In other words, changing the perspective increased the integrative processing of the relevant sentences.

Probability of skipping. A significant main effect of relevance $\left[F(1,58)=46.60, M S_{\mathrm{e}}=0.04, \eta^{2}=.45\right]$ indicates that a repetition benefit of $8.5 \%$ was observed for the irrelevant sentences and a repetition cost of 5.7\% for the relevant sentences. However, the main effect of relevance was modulated by two interactions. The relevance $\times$ text interaction $\left[F(1,58)=5.52, M S_{e}=0.04\right.$, $\left.\eta^{2}=.09\right]$ suggests that the magnitude of the repetition effect for relevant and irrelevant sentences was different in the two texts. In the LPK text, we observed a 7\% repetition benefit for the irrelevant sentences $[t(29)=-3.72$, $S E=0.02]$ but a nonsignificant $3 \%$ repetition cost for the relevant sentences $(t<2$; see Figure 2). In the HPK text, there was a $10 \%$ repetition benefit in the processing of the irrelevant sentences $[t(29)=-2.90, S E=0.04]$ and a $9 \%$ repetition cost in the processing of the relevant sentences $[t(29)=2.67, S E=.04]$. Second, a significant relevance $\times$ position interaction was observed $[F(1,85)=$ $\left.9.17, M S_{\mathrm{e}}=0.02, \eta^{2}=.14\right]$, indicating that the difference between the relevant and the irrelevant sentences in the magnitude of the repetition effect varied across the word positions. A significant processing benefit was observed for the perspective-irrelevant sentences in the middle $(10 \%)$ and in the end $(11 \%)$ of the sentences [smallest $t(59)=-4.75, S E=0.02$, in the end]. A significant repetition cost for the perspective-relevant sentences was evident in the middle $(6 \%)$ and in the end $(8 \%)$ of the sentences [smallest $t(59)=3.11, S E=0.03$, in the end].

To sum up, changing the reading perspective between the second and the third readings brought about the following processing effects, as reflected in word skipping. A significant repetition benefit was observed for irrelevant sentences in both texts. A repetition cost for the relevant sentences was observed only in the HPK text; the lack of the processing cost for the relevant sentences in the LPK text is probably a consequence of relatively little skipping in the LPK text during the second reading. The magnitude of the repetition benefit for the irrelevant sentences and the cost for relevant sentences grew from the beginning to the end of the sentence, being greatest in the sentence end. This finding suggests that during the third reading, the selectivity in processing increases toward the sentence end.

Probability of regression. In the third reading, we observed for the probability of regression a main effect of relevance $\left[F(1,58)=53.51, M S_{\mathrm{e}}=0.04, \eta^{2}=.48\right]$, a relevance $\times$ position interaction $\left[F(2,94)=16.66, M S_{\mathrm{e}}=\right.$

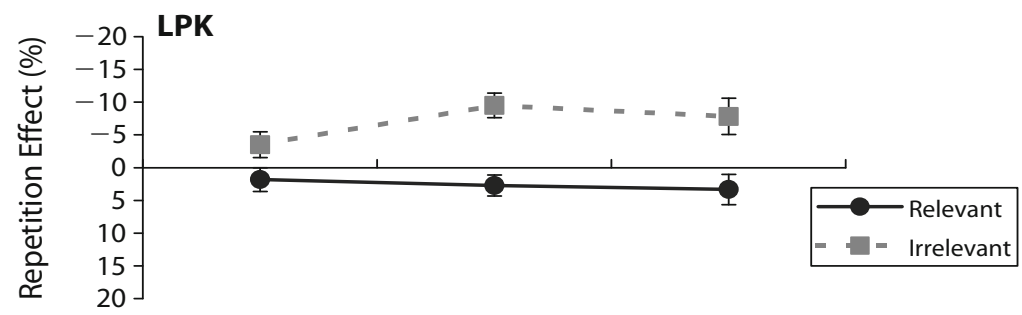

Beginning Middle End

Word Position

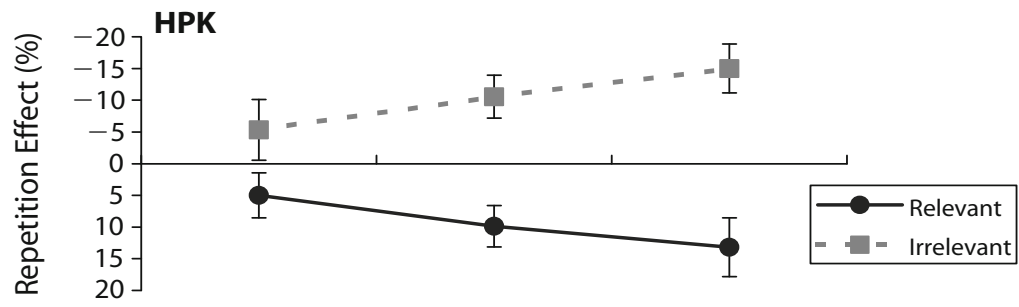

Beginning Middle End

Word Position

Figure 2. Mean repetition effects in skipping rate (in percentages) in the third reading for the relevant and irrelevant text segments as a function of the position of the word in the sentence (error bars represent $1 S E$ above and below the mean). Different panels represent data for different texts: LPK, low prior knowledge text; HPK, high prior knowledge text. Scale is reversed because negative values reflect larger repetition benefit. 

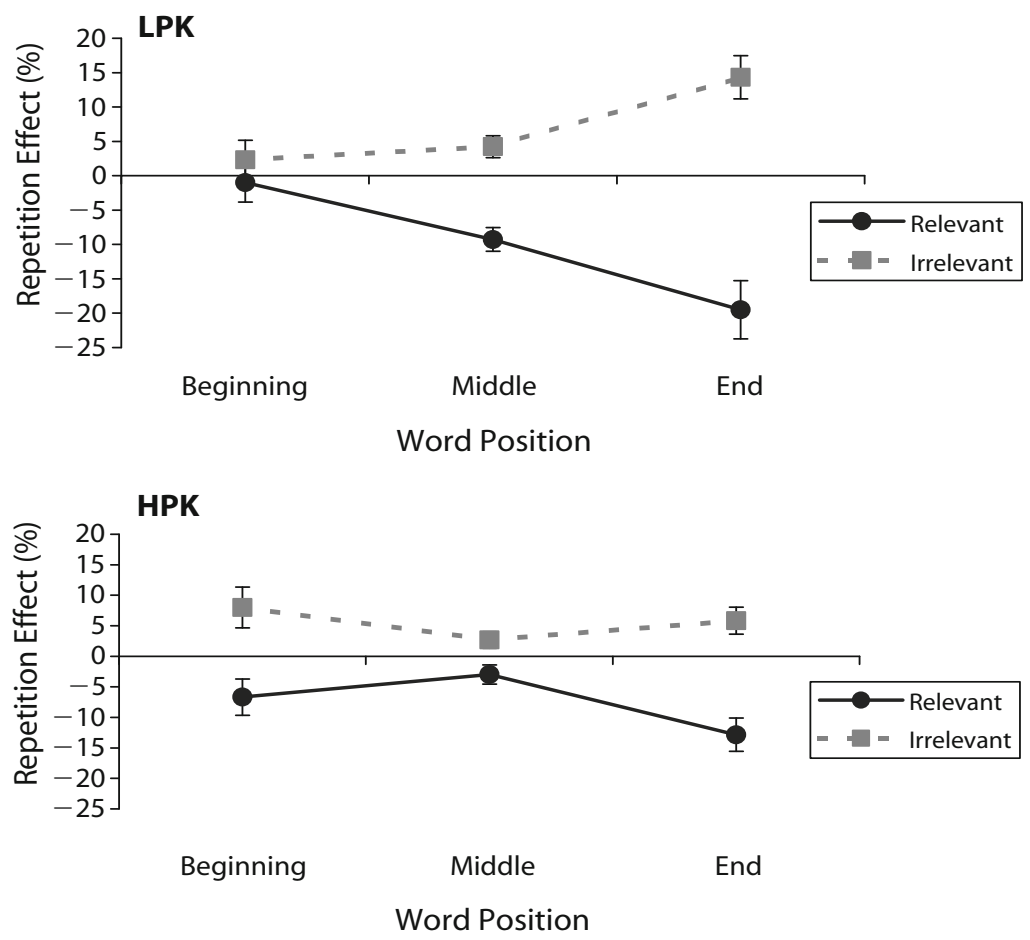

Figure 3. Mean repetition effects in probability of regression (\%) in the third reading for the relevant and irrelevant text segments as a function of the position of the word in the sentence (error bars represent $1 S E$ above and below the mean). Different panels represent data for different texts: LPK, low prior knowledge text; HPK, high prior knowledge text.

$\left.0.02, \eta^{2}=.22\right]$, and a significant three-way interaction between relevance, position, and text $[F(2,94)=8.13$, $\left.M S_{\mathrm{e}}=0.02, \eta^{2}=.12\right]$. The main effect of relevance suggests a repetition cost for relevant sentences (a 9\% increase in the probability of regression) and a repetition benefit $(6.5 \%)$ for the irrelevant sentences. The interaction between relevance and word position indicates that the repetition effects for both the relevant and the irrelevant sentences were greatest in the sentence end. To examine the three-way interaction, separate ANOVAs were computed for the two texts. In the LPK text, there was a significant relevance $\times$ position interaction $\left[F(2,47)=19.93, M S_{\mathrm{e}}=\right.$ $\left.0.02, \eta^{2}=.41\right]$. As is evident from Figure 3, readers regressed less in the irrelevant sentences (repetition benefit of $4 \%$ for the middle and $14 \%$ for the final position) and more in the relevant sentences (repetition cost of $9 \%$ for the middle and $20 \%$ for the final position) on the third reading, which effects were most robust for the sentencefinal position [smallest $t(29)=2.65, S E=0.02$, for the sentence-middle position of the irrelevant sentences]. In the HPK text, the relevance $\times$ position interaction proved also significant $\left[F(1,43)=4.07, M S_{\mathrm{e}}=0.02, \eta^{2}=.12\right]$. However, the nature of the interaction was quite different from that in the LPK text. As is shown in Figure 3, there was a significant repetition benefit for the irrelevant sentences (8\% in the beginning and $6 \%$ in the end position) and a repetition cost for the relevant sentences $(7 \%$ in the beginning and $13 \%$ in the end) [smallest $t(29)=-2.25$,
$S E=0.03$, in the sentence-initial position of the relevant sentences]. In other words, the repetition benefit for irrelevant sentences peaked earlier (in the sentence-initial position) than did the repetition cost for relevant sentences (in the sentence-final position).

In sum, the analysis of the probability of regression demonstrated an interesting difference between the LPK and the HPK texts. In addition to affecting the need for sentence wrap-up processing in the sentence end in both texts, a change in the reading perspective affected the probability of launching an early regression from the beginning of the irrelevant sentences in the HPK text. This pattern suggests that the reading perspective exerts an immediate effect in the HPK text and a relatively later effect in the LPK text.

\section{Recall}

The recall protocols were analyzed for the amount of relevant and irrelevant text information correctly recalled. The participant was credited 1 point for each target sentence for which he or she correctly recalled the gist (no verbatim recall of the target sentence was required). In order to define the interrater reliability, two independent raters scored 10 protocols. The mean agreement was very high (95\%); thus, only one rater scored the rest of the protocols. The data for the same 60 participants contributing to the fixation time measures were included in the analyses. Two separate 2 (text: HPK vs. LPK) $\times 2$ (perspec- 
tive relevance: relevant vs. irrelevant) repeated measures ANOVAs were computed for the first and second recall. Text was entered as a between-participants factor, and perspective relevance was entered as a within-participants factor.

We expected that readers would have superior memory for relevant (in comparison with irrelevant) text information. We also expected that after rereading the text from a different perspective, they would mostly add relevant facts to their recall protocol.

The recall results are presented in Table 4. The participants recalled more relevant than irrelevant target information during the first recall $\left[F(1,58)=48.36, M S_{\mathrm{e}}=\right.$ $\left.3.78, p<.001, \eta^{2}=.46\right]$. After the perspective change, the participants added to their protocols more information that became relevant after the perspective change than initially relevant information $\left[F(1,58)=67.20, M S_{\mathrm{e}}=2.29\right.$, $\left.p<.001, \eta^{2}=.54\right]$. In other words, the recall data suggest that the reading perspective strongly determined the memory for text: The perspective-relevant text information was encoded to memory much better than the perspectiveirrelevant text information. After the perspective change, the participants upgraded their memory representation for previously irrelevant text information so that their recall now matched the level of recall for the initially relevant text information. At the first recall attempt, the participants recalled an average of 6.22 relevant target sentences and 3.75 irrelevant target sentences (across the two texts). In the second recall attempt, the readers added to their recall protocols an average of 2.90 originally irrelevant target sentences that became relevant after the perspective change, resulting in a mean recall of $3.75+2.90=6.65$ for the relevant target sentences.

\section{DISCUSSION}

The present study examined the detailed time course and nature of the perspective effects in text comprehension. The first question we explored was whether reading perspective exerts its influence at an early phase of target sentence processing or whether it mainly increases the need for later integrative processing. The results suggest that the adopted reading perspective modifies the scanning strategies used during the first-pass reading of text sentences; it is thus capable of exerting its influence already at the construction phase of comprehension (cf. Kintsch, 1998). There were two pieces of evidence supporting an early influence of reading perspective. First, gaze duration was longer in relevant than in irrelevant sentences already for the sentence-initial words. Second, readers also tended to skip over words in irrelevant sentences. In addition to these early processing effects, reading perspective increased the need for later integrative processing, as was shown by longer gaze durations for sentence-final words in the relevant target sentences than in the irrelevant target sentences and by an increased number of regressions made in relevant than in irrelevant sentences. More generally speaking, these results suggest that reading perspective guides readers' visual attention in a selective manner (Anderson, 1982) and defines what information is processed to a deeper level and integrated to the developing memory representation.

On the basis of our previous results (Kaakinen et al., 2003), the timing of the perspective effect was assumed to be modulated by prior knowledge: When readers have no or little background knowledge about the text contents, the perspective effects were expected to manifest later in the processing time course. Indeed, in the LPK text, the bulk of the perspective effect was relatively late, since it showed up particularly strongly at the sentencefinal position. In other words, the processing of relevant sentences differed from that of irrelevant sentences at the stage of wrapping up and rehearsing the sentence meaning before moving on to the next sentence. In the HPK text, on the other hand, the perspective effect was spread more evenly throughout the sentence. In other words, it was clearly present prior to reaching the sentence end. This is shown in the probability of regression for which a reliable perspective effect was observed already in the sentence beginning. We also obtained evidence indicating that the late effect of reading perspective (as indexed by the probability of regression from the sentence end) was larger in the LPK than in the HPK text, whereas the early perspective effect (as indexed by word skipping during the first-pass reading) was greater in the HPK than in the LPK text. In other words, when the readers had minimal or no prior knowledge about the text contents, they had to devote extra effort at sentence boundaries to encode relevant information to memory, whereas when they had ample prior knowledge available, they were able to adopt a selective processing strategy and skip over words in the irrelevant sentences. In general, these results demonstrate that reading perspective is more readily utilized to guide online processing of text with familiar contents than of that with unfamiliar contents.

The second question of interest was how readers utilize the reading perspective when they reread a text. According to one possibility, readers divide their visual attention relatively evenly between relevant and irrelevant text information during the first reading but then switch to a

Table 4

Mean Recall (Maximum $=10$ ) of the Relevant and Irrelevant Information in the Low and High Prior Knowledge Texts for the First Recall (i.e., After Two Readings From the Same Perspective) and the Additional Recall (After the Third Reading, Which Was Preceded by a Perspective Shift)

\begin{tabular}{|c|c|c|c|c|}
\hline \multirow[b]{2}{*}{ Target Sentence } & \multicolumn{2}{|c|}{$\begin{array}{c}\text { Low Prior } \\
\text { Knowledge } \\
\text { Text }\end{array}$} & \multicolumn{2}{|c|}{$\begin{array}{c}\text { High Prior } \\
\text { Knowledge } \\
\text { Text }\end{array}$} \\
\hline & $M$ & $S E$ & $M$ & $S E$ \\
\hline \multicolumn{5}{|c|}{ First Recall } \\
\hline $\begin{array}{l}\text { Relevant } \\
\text { Irrelevant }\end{array}$ & $\begin{array}{l}6.37 \\
3.43\end{array}$ & $\begin{array}{l}0.33 \\
0.42\end{array}$ & $\begin{array}{l}6.07 \\
4.07\end{array}$ & $\begin{array}{l}0.43 \\
0.39\end{array}$ \\
\hline \multicolumn{5}{|c|}{ Additional Recall } \\
\hline $\begin{array}{l}\text { Relevant } \\
\text { Irrelevant }\end{array}$ & $\begin{array}{l}3.10 \\
0.67\end{array}$ & $\begin{array}{l}0.33 \\
0.15\end{array}$ & $\begin{array}{l}2.70 \\
0.60\end{array}$ & $\begin{array}{l}0.35 \\
0.21\end{array}$ \\
\hline
\end{tabular}

Note-Means for the additional recall represent the amount of information that the participants added to their recall protocols after the third reading of the text. 
more strategic processing mode during the second reading by paying particular attention to perspective-relevant information. Alternatively, if readers devote a lot of attention to relevant information during the initial reading, its processing may be significantly facilitated during repeated reading, resulting in a smaller perspective effect. We also predicted that, similarly to background knowledge, prior exposure to text would shift the time course of the observed perspective effects. When participants were reading the same text for the second time, perspective effects were expected to show up earlier in the processing time course than during the initial reading.

None of the predictions above were confirmed. We observed that when our experimental texts were immediately reread from the same reading perspective, the pattern of eye fixations changed surprisingly little between the first and second readings. The only significant finding (apart from there being a general speeding up of reading reflected in all eye movement measures) was that repetition particularly affected the processing done at the sentence end, in the form of shorter gaze durations on the sentence-final words and a reduced number of regressions made from the sentence end to a previous part of the target sentence. These findings indicate that repetition especially reduced the need for integrative processing done at the sentence end. These results are in accordance with the study of Hyönä and Niemi (1990), who examined the effect of repeated reading on eye movement parameters for sentences that were rated as important or unimportant in the text (no specific reading perspective was assigned prior to reading). Repetition decreased the total fixation time, the average fixation duration, the average saccade length, the number of progressive fixations, and the number of regressions in the sentences. More important, the magnitude of the importance effect did not change across three readings of the same text, but pertinent sentences consistently received more visual attention than did less pertinent sentences during all the readings - a pattern compatible with that observed in the present study for the difference between perspective-relevant and perspectiveirrelevant sentences. Taken together, these two studies suggest that perceived importance and assigned relevance of text information are capable of exerting a solid and stable effect on guiding the eyes and visual attention through the text.

We also examined the nature of the memory representation constructed for relevant and irrelevant text information by asking the readers to switch perspective before the third reading of the text. Perspective switch swapped the relevance of the target sentences: Information that was initially irrelevant became relevant after the perspective change, and information that was initially relevant now became irrelevant. When the same text was read a third time, but from a different reading perspective, we observed in all our eye movement parameters a repetition benefit for irrelevant sentences and a repetition cost for relevant sentences. These data suggest that during the first two readings, the readers constructed a more elaborate episodic memory representation for the relevant than for the irrelevant sentences (see also Baillet \& Keenan, 1986; Kaaki- nen, Hyönä, \& Keenan, 2001). Had they properly encoded to memory the contents of the irrelevant sentences, they would not have needed the extra time to reprocess them as perspective relevant. This extra effort is assumed to be spent in wrapping up the meaning of the sentence and encoding its main contents to memory. This view is further supported by the findings that the extra processing cost was particularly evident in the eye movement measures for the sentence-final position, which presumably index sentence wrap-up processing. The recall data corroborate these findings. The participants had better memory for relevant than for irrelevant text information, and after the perspective change, they added more relevant than irrelevant information to their recall protocols.

We next will discuss the implications of the present results to theories of eye guidance in reading. As may be recalled, we observed that words in the perspectiverelevant sentences were read with longer gaze durations, were skipped less often, and were read with more regressions than were perspective-irrelevant sentences. The most prominent effects of reading perspective were observed as increased sentence wrap-up processing (Just \& Carpenter, 1980; Rayner et al., 2000) in the end of the relevant sentences. These findings held even when the same text was reread from the same perspective. Thus, reading perspective exerts a global effect on readers' eye movements, since both temporal (when to terminate a fixation) and spatial (where to fixate next) aspects of eye behavior were affected. What is also noteworthy is that reading perspective not only influences the second-pass reading and sentence wrap-up processing, but also is capable of affecting the first-pass reading, as indexed by the gaze duration and skipping data. The effect of reading perspective may be considered a type of context effect, where the perspective given to the reader prior to reading determines what is relevant versus irrelevant in the text. Thus, on a general level, all eye guidance models that take contextual constraints into account are, at least in principle, in a position to account for the observed effects. Of the existing ones, the E-Z Reader model (Reichle, Rayner, \& Pollatsek, 2003) and SWIFT (Engbert, Longtin, \& Kliegl, 2002) are equipped to account for context effects. At present, they are geared only to model effects related to contextual predictability within single sentences, but they probably can be adjusted to account for higher order context effects, such as perspective effects. It should be noted, however, that perspective (ir)relevance is not equivalent to contextual predictability and that the way these two factors influence online text processing is probably different. This is evident in the finding that even though readers had constructed a good memory representation of the relevant text contents during the first reading of the text, the perspective effect obtained during the second reading did not manifest earlier in the eye movement record. This would have been expected, particularly for the HPK text, if reading perspective worked similarly to contextual predictability.

In closing, the present results support a framework for perspective-driven text comprehension (Kaakinen \& Hyönä, in press), which assumes a crucial role of prior 
knowledge in how readers allocate visual attention during reading. In our framework, it is assumed that the perspective adopted by the reader defines the standards of coherence for the evolving memory representation (van den Broek, Risden, \& Husebye-Hartmann, 1995). Since higher standards apply to relevant than to irrelevant text information, readers try to gain a more comprehensive memory for perspective-relevant text information (Kaakinen \& Hyönä, 2005), whereas a relatively superficial memory representation is considered sufficient for perspectiveirrelevant information. Deployment of overt visual attention is adjusted to meet the standards of coherence.

According to the framework (Kaakinen \& Hyönä, in press), perspective instructions given prior to reading activate perspective-relevant prior knowledge in the reader's knowledge base. During the course of reading, text input is constantly interpreted in the light of the reading perspective, relevant background knowledge (if available), and already read text information. Availability of prior knowledge modulates the way visual attention is allocated in the text. When perspective-relevant text information is encountered, the available prior knowledge resonates with the text input, allowing a quick recognition of the text information as relevant. Consequently, perspectiverelevant text information is easily incorporated into the developing text representation, and the high standards of coherence are met without much additional effort. Perspective-irrelevant information, on the other hand, is only superficially processed: Even though readers may read through the irrelevant sentences in order to be able to determine their irrelevance, readers do not attempt to integrate irrelevant information into the developing memory representation, and they proceed in the text relatively quickly. However, if the reader does not have relevant prior knowledge available, recognizing the incoming text information as perspective relevant (or irrelevant) may not be readily accomplished. Moreover, building the necessary links to incorporate the relevant text information into the developing memory representation requires extra attentional effort. Thus, readers may decide to reread the relevant sentence before moving on in the text. Or they may stop at the sentence end to allow additional time for integrative processing.

The proposed framework (Kaakinen \& Hyönä, in press) is not inconsistent with other existing models of text comprehension (cf. Kintsch, 1998); its main contribution is that it specifies the role of reading goal or perspective in text comprehension.

\section{AUTHOR NOTE}

Parts of the data were presented at the 45th Annual Meeting of the Psychonomic Society, Minneapolis, 2004. J.H. was supported by the Academy of Finland. Correspondence concerning this article should be addressed to J. K. Kaakinen, Department of Psychology, University of Turku, FIN-20014 Turku, Finland (e-mail: johanna.kaakinen@utu.fi).

\section{REFERENCES}

ANDERSON, R. C. (1982). Allocation of attention during reading. In A. Flammer \& W. Kintsch (Eds.), Discourse processing (pp. 292305). Amsterdam: North-Holland.
Anderson, R. C., \& Pichert J. W. (1978). Recall of previously unrecallable information following a shift in perspective. Journal of Verbal Learning \& Verbal Behavior, 17, 1-12.

Anderson, R. C., Pichert, J. W., \& Shirey, L. L. (1983). Effects of the reader's schema at different points in time. Journal of Educational Psychology, 75, 271-279.

Baillet, S. D., \& Keenan, J. M. (1986). The role of encoding and retrieval processes in the recall of text. Discourse Processes, 9, 247268.

Blanchard, H. E., \& Iran-Nejad, A. (1987). Comprehension processes and eye movement patterns in the reading of surprise-ending stories. Discourse Processes, 10, 127-138.

Engbert, R., Longtin, A., \& Kliegl, R. (2002). A dynamical model of saccade generation in reading based on spatially distributed processing. Vision Research, 42, 621-636.

ERICsSON, K. A., \& Kintsch, W. (1995). Long-term working memory. Psychological Review, 102, 211-245.

Goetz, E. T., Schallert, D. L., Reynolds, R. E., \& Radin, D. I. (1983). Reading in perspective: What real cops and pretend burglars look for in a story. Journal of Educational Psychology, 75, 500-510.

HYöNÄ, J. (1995). An eye movement analysis of topic-shift effect during repeated reading. Journal of Experimental Psychology: Learning, Memory, \& Cognition, 21, 1365-1373.

HYÖNÄ, J., \& NIEMI, P. (1990). Eye movements during repeated reading of a text. Acta Psychologica, 73, 259-280.

Just, M. A., \& Carpenter, P. A. (1980). A theory of reading: From eye fixations to comprehension. Psychological Review, 87, 329-354.

KAAKINEN, J. K., \& HyÖNÄ, J. (2005). Perspective effects on expository text comprehension: Evidence from think-aloud protocols, eyetracking and recall. Discourse Processes, 40, 239-257.

KAAKINEN, J. K., \& HyÖNÄ, J. (in press). Perspective-driven text comprehension. Applied Cognitive Psychology.

KaAkinen, J. K., Hyönä, J., \& Keenan, J. M. (2001). Individual differences in perspective effects on text memory. Current Psychology Letters: Behaviour, Brain \& Cognition, 5, 21-32.

KaAkinen, J. K., Hyönä, J., \& Keenan, J. M. (2002). Perspective effects on on-line text processing. Discourse Processes, 33, 159-173.

KaAkinen, J. K., Hyönä, J., \& Keenan, J. M. (2003). How prior knowledge, WMC, and relevance of information affect eye fixations in expository text. Journal of Experimental Psychology: Learning, Memory, \& Cognition, 29, 447-457.

KINTSCH, W. (1988). The role of knowledge in discourse comprehension: A construction-integration model. Psychological Review, 95, 163-182.

Kintsch, W. (1998). Comprehension: A paradigm for cognition. Cambridge: Cambridge University Press.

Kintsch, W., \& FranzKe, M. (1995). The role of background knowledge in the recall of a news story. In R. F. Lorch \& E. J. O'Brien (Eds.), Sources of coherence in reading (pp. 321-333). Hillsdale, NJ: Erlbaum.

Kintsch, W., Patel, V. L., \& Ericsson, K. A. (1999). The role of longterm working memory in text comprehension. International Journal of Psychology in the Orient, 42, 186-198.

Laine, M., \& Virtanen, P. (1999). WordMill Lexical Search Program. Turku, Finland: University of Turku, Centre for Cognitive Neuroscience.

Levy, B. A., Campsall, J., Browne, J., Cooper, D., Waterhouse, C., \& WiLsON, C. (1995). Reading fluency: Episodic integration across texts. Journal of Experimental Psychology: Learning, Memory, \& Cognition, 21, 1169-1185.

Levy, B. A., \& Kirsner, K. (1989). Reprocessing text: Indirect measures of word and message level processes. Journal of Experimental Psychology: Learning, Memory, \& Cognition, 15, 407-417

McNamara, D. S., \& KinTsch, W. (1996). Learning from texts: Effects of prior knowledge and text coherence. Discourse Processes, 22, 247-288.

MiLLIS, K. K., \& KING, A. (2001). Rereading strategically: The influences of comprehension ability and a prior reading on the memory for expository text. Reading Psychology, 22, 41-65.

Millis, K. K., KING, A, \& KIM, H. J. (2000). Updating situation models from descriptive texts: A test of the situational operator model. Discourse Processes, 30, 201-236.

Millis, K. K., \& Simon, S. (1994). Rereading scientific texts: Changes 
in resource allocation. In H. van Oostendorp \& R. A. Zwaan (Eds.) Naturalistic text comprehension (pp. 115-133). Norwood, NJ: Ablex.

Millis, K. K., Simon, S., \& tenBroek, N. S. (1998). Resource allocation during the rereading of scientific texts. Memory \& Cognition, 26, 232-246.

MorAvCSIK, J. E., \& KINTSCH, W. (1995). Writing quality, reading skills, and domain knowledge as factors in text comprehension. In J. M. Henderson, M. Singer, \& F. Ferreira (Eds.), Reading and language processing (pp. 232-246). Mahwah, NJ: Erlbaum.

Pichert, J. W., \& Anderson, R. C. (1977). Taking different perspectives on a story. Journal of Educational Psychology, 69, 309-315.

RANEY, G. E. (2003). A context-dependent representation model for explaining text repetition effects. Psychonomic Bulletin \& Review, 10, $15-28$.

Rayner, K., Kambe, G., \& Duffy, S. A. (2000). The effect of clause wrap-up on eye movements during reading. Quarterly Journal of Experimental Psychology, 53A, 1061-1080.

Reichle, E. D., Rayner, K., \& Pollatsek, A. (2003). The E-Z Reader model of eye movement control in reading: Comparisons to other models. Behavioral \& Brain Sciences, 26, 445-526.

Shebilske, W. L., \& Fisher, D. F. (1981). Eye movements reveal components of flexible reading strategies. In M. L. Kamil (Ed.), 30th yearbook of the National Reading Conference (pp. 51-56). Washington, DC: The National Reading Conference.
Stine-Morrow, E. A. L., Gagne, D. D., Morrow, D. G., \& DeWall, B. H. (2004). Age differences in rereading. Memory \& Cognition, 32, 696-710.

van den Broek, P., Risden, K., \& Husebye-Hartmann, E. (1995) The role of readers' standards for coherence in the generation of inferences during reading. In R. F. Lorch, Jr., \& E. J. O’Brien (Eds.), Sources of coherence in text comprehension (pp. 353-373). Hillsdale, NJ: Erlbaum.

Voss, J. F., \& Silfies, L. N. (1996). Learning from history text: The interaction of knowledge and comprehension skill with text structure. Cognition \& Instruction, 14, 45-68.

\section{NOTE}

1. There were 9.50 words and 71.70 characters in the diarrhearelevant, 8.80 words and 70.20 characters in the flu-relevant, 9.40 words and 76.40 characters in the typhus-relevant, and 9.20 words and 71.90 characters in the trigeminusneuralgy-relevant target sentences. The logarithmic lemma frequencies of the words appearing in the sentences were 8.12 in the diarrhea-relevant, 7.78 in the flu-relevant, 8.10 in the typhus-relevant, and 7.73 in the trigeminusneuralgy-relevant target sentences. Thus, the target sentence sets were matched in length and word frequency between the relevant and the irrelevant sets and across the two texts.

\footnotetext{
The following is an example paragraph from the rare diseases (LPK) text (trigeminusneuralgy-related target sentences are in boldface, typhus-related sentences are in italic). In the actual experiment, the target sentences were not boldfaced or italicized. The subheading ("Treatment") preceding the paragraph was presented in boldface.

\section{Treatment}

Most of the diseases are treatable, or at least their symptoms can be somehow relieved. Typhus is nearly extinct because there is an efficient treatment for it. The disease can be treated with strong antibiotics. Tetracyclic antibiotics are very effective. The best way to treat scleroderma is to cover the sensitive skin from injuries and cold. Blood circulation can be enhanced with medication that expands the vessels. Also, stopping smoking enhances circulation. However, there is no actual cure for scleroderma. The metabolic dysfunction in cystic fibrosis can be balanced with pancreatic enzymes. In addition, regular, moderate exercise helps to remove viscous mucus from the lungs. Treatment of trigeminusneuralgy is a lot more difficult. Over-thecounter drugs do not help nearly at all. Most-often-used medication is a combination of depression and epilepsy drugs. In addition, it is possible to treat the pain with a surgery of the tactile nerves in the face. The nerve can be cut or it can be injected with glycerol or alcohol. The problem is that one side of the face goes numb, which might be uncomfortable.

Below is an example paragraph from the common diseases (HPK) text (diarrhea-related target sentences are in boldface, flu-related sentences are in italic). In the actual experiment, the target sentences were not boldfaced or italicized. The subheading ("In milder diseases home treatment is enough") preceding the paragraph was presented in boldface.

\section{In Milder Diseases Home Treatment Is Enough}

The most common infectious diseases can be defeated by home treatment. The itching caused by chicken pox can be relieved by applying menthol oil on the skin. In diarrhea it is very important to drink a lot and to compensate for the lost liquids and minerals. The best home treatment is to drink a lot of liquids-for example, blueberry soup or fruit juices. After the diarrhea eases out, it is advisable to slowly transfer from liquids to solid food. Fortunately the diarrhea usually lasts only a few days and cures on its own. The best home treatment for flu, on the other hand, is rest. One should avoid strenuous exercise during flu because it may cause sequelae. It is also good to drink hot beverages, for example hot juice. In addition to rest and hot drinks, inhaling steam may help the condition of a flu patient.
}

APPENDIX

(Manuscript received December 19, 2005;

revision accepted for publication September 18, 2006.) 